ICMB21

\title{
Assessing the performance of Internal Wall Insulation considering transient conditions
}

\author{
Hannah, N. ${ }^{\mathrm{a}}$, Marincioni, V. ${ }^{\mathrm{a}}$, Chalabi, Z. ${ }^{\mathrm{a}}$, Altamirano-Medina, H. ${ }^{\mathrm{a}}$ \\ a UCL Institute for Environmental Design and Engineering, London, UK
}

\begin{abstract}
Recent research suggests that every insulation material undergoes failure in varying degrees of severity. Based on data derived from specified mould risk failure criteria, this study developed a novel equation to quantify the performance of insulation materials/systems following such transient conditions. Transient conditions performance (TCP) was quantified using the period after exceeding the risk criteria, the duration of exceedance and recovery time. The current formulation does not readily distinguish between materials based on their ability to cope with transient conditions. Further research was initiated to incorporate more diverse variables with which to expand the specificity of the TCP quantification. Peer-review under the responsibility of the organizing committee of the ICMB21.
\end{abstract}

Keywords: Internal Wall Insulation; Interstitial Condensation; Mould/Fungal Risk Assessment; Insulation System Transient Conditions

\begin{tabular}{|ll}
\hline Nomenclature & \\
IWI & Internal wall insulation \\
MW-VCL & Mineral wool with a vapour control layer \\
PIR & Polyisocyanurate \\
\hline
\end{tabular}

\section{Introduction}

1.1 Background

The current UK building standards regulating mould risk, Approved Document F (ADF) [1], do not provide clear guidance on what is to be done if a material fails its mould risk assessment criteria. In a recent study [2], all insulation materials used as internal wall insulation were predicted to exceed the ADF mould criteria for as high as $80 \%$ of the time. Failure within IWI can go unnoticed for long periods of time; even if recognized, it is unrealistic to replace IWI at the earliest sign of failure. Therefore, material performance exceeding mould growth risk criteria must be better understood to improve and inform IWI material/system selection at the design stage for optimized structural lifespan.

\subsection{Aim and Objectives}

This paper aims to define the performance of insulation materials during transient conditions for the purpose of material comparison and longevity projection. Besides, the study proposes research to validate the idea of a transient condition quantification to further assess and compare materials post RH exceedances.

\section{Methodology}

\subsection{Defining and quantifying transient conditions}

Coping with transient conditions was defined as the ability of an IWI material/system to return from favourable conditions for mould growth, where the risk of mould development is greater than $0 \%$ due to accumulated moisture, as defined in ADF. The quantification of these transient conditions was built upon previous mathematical work by Chalabi [3], which calculated the average period between breaches of critical RH limits (time passing criteria exceedance, $\mathrm{t}_{\mathrm{P}}$ ). This term was then divided by one half of the average time in exceedance of the criteria - termed recovery time, $t_{\mathrm{R}}$.

Temperature and RH data from Marincioni [2] were post-processed in MS Excel to determine the number of hours exceeding the moisture criteria ${ }^{1}$ as well as the frequency of criteria exceedance for MW-VCL and PIR across each scenario described in [2] (combinations of wall type, orientation, U-value, and location). The outputs were used to calculate the proportion of time passing (\%) and score the materials for their transient condition performance (TCP) through $t_{\mathrm{p}} / \mathrm{t}_{\mathrm{R}}$. Twentyfour scenarios of data were used to carry out the resilience performance comparison.

\section{Results \\ 3.1 Comparison by TCP as defined}

Although significant performance differentiation through TCP scores was desired, the twenty-four scenarios returned nearly identical percent passing and TCP between the two materials (MW-VCL and PIR), given otherwise constant scenarios (Table 1). There was far more variability when considering other factors relating to the scenarios themselves, rather than material performance, demonstrated through the comparison of MW-VCL to PIR in the same scenario, versus comparing a material's performance against itself in other scenarios. The results vary drastically more between scenarios than between materials. Only $3 / 24$ scenarios differed between the two materials by more than $1.5 \%$ (outlined in bold within Table 1 ). However, it is unclear whether these differences of $\sim 4, \sim 11$, and $\sim 23 \%$ are anomalies in the simulation or a significant performance differentiation between the two materials. More analysis would be necessary to validate, qualify or reject these

\footnotetext{
${ }^{1}$ Criteria based on new critical RH proposed in [4].
} 


\section{ICMB21}

results fully. Variations within the greater IWI systems (wall type, orientation, U-value, location) dictating notable change are discussed in detail in [2].

Table 1 Output from the 48-scenario analysis run on MW-VCL and PIR materials. Percent passing and TCP score are calculated for each scenario. The colourcoding visualizes various outcomes (red, no passing (always breaching critical criteria limits) and TCP of 0; yellow, 0.01-49.9\% passing and 0.1-0.99 TCP; light green, 50-75\% passing and 1-2 TCP; dark green, 75-99.9\% passing and >2TCP; no fill, $100 \%$ passing and no TCP score (-)).

\begin{tabular}{|c|c|c|c|c|c|c|c|c|c|c|}
\hline \multirow{2}{*}{\multicolumn{3}{|c|}{$\underline{\text { Scenario Variables }}$}} & \multicolumn{4}{|c|}{ MW-VCL } & \multicolumn{4}{|c|}{ PIR } \\
\hline & & & \multicolumn{2}{|c|}{ Bristol Centre } & \multicolumn{2}{|c|}{$\underline{\text { Clifton }}$} & \multicolumn{2}{|c|}{$\underline{\text { Bristol Centre }}$} & \multicolumn{2}{|c|}{$\underline{\text { Clifton }}$} \\
\hline Wall Type & Orientation & U-value & $\%$ Passing & $\mathrm{TCP}$ & $\%$ Passing & TCP & $\%$ Passing & TCP & $\%$ Passing & $\mathrm{TCP}$ \\
\hline \multirow{4}{*}{ Brick } & \multirow{2}{*}{$\mathrm{N}$} & 0.4 & 28.85 & 0.54 & 100.00 & - & 27.55 & 0.51 & 100.00 & - \\
\hline & & 0.6 & 55.50 & 1.66 & 100.00 & - & 55.47 & 1.66 & 100.00 & - \\
\hline & \multirow{2}{*}{ SW } & 0.4 & 29.83 & 0.57 & 0.00 & 0.00 & 41.38 & 1.06 & 0.00 & 0.00 \\
\hline & & 0.6 & 50.45 & 1.36 & 0.00 & 0.00 & 49.87 & 1.33 & 0.00 & 0.00 \\
\hline \multirow{4}{*}{$\begin{array}{c}\text { Porous } \\
\text { Stone }\end{array}$} & \multirow{2}{*}{$\mathrm{N}$} & 0.4 & 0.00 & 0.00 & 58.08 & 1.85 & 0.00 & 0.00 & 57.91 & 1.83 \\
\hline & & 0.6 & 20.86 & 0.35 & 100.00 & - & 21.15 & 0.36 & 100.00 & - \\
\hline & \multirow{2}{*}{ SW } & 0.4 & 0.00 & 0.00 & 0.00 & 0.00 & 0.00 & 0.00 & 0.00 & 0.00 \\
\hline & & 0.6 & 0.00 & 0.00 & 0.00 & 0.00 & 0.00 & 0.00 & 0.00 & 0.00 \\
\hline \multirow{4}{*}{$\begin{array}{c}\text { Non-Porous } \\
\text { Stone }\end{array}$} & \multirow{2}{*}{$\mathrm{N}$} & 0.4 & 100.00 & - & 100.00 & - & 100.00 & - & 100.00 & - \\
\hline & & 0.6 & 100.00 & - & 100.00 & - & 100.00 & - & 100.00 & - \\
\hline & \multirow{2}{*}{ SW } & 0.4 & 100.00 & - & 77.57 & 5.76 & 100.00 & - & 100.00 & - \\
\hline & & 0.6 & 100.00 & - & 100.00 & - & 100.00 & - & 100.00 & - \\
\hline
\end{tabular}

\subsection{Detailing favourable conditions using a summative equation}

The initial representation of the transient conditions failed to justify its usefulness as a material performance measurement scale. A new quantification of TCP redirects the focus from a passing/exceedance perspective to an equation that comprises various additive terms based on ideas from current risk assessment literature on factors shown to affect the viability of fungi.

The alternative quantification is intended to distil many distinct factors that affect the likelihood of fungal activity down into an easy-to-use equation that requires basic inputs and produces a single metric with which to compare insulation material/system performance. Currently, the transient conditions are largely ignored by the published regulations, lumping them into the category of breaching critical RH criteria, and therefore suggesting fungal activity which may, in reality, not be present. As the initial representation for the transient condition performance was considerably oversimplified, a robust equation was developed that is transparent yet, specific enough to improve the understanding of IWI systems.

Spurred from the above work, the terms incorporated into the equation were compiled in a novel fashion, to produce a single-metric outcome by organizing the variables into a sum of dimensionless terms. These terms included the material performance relative to the critical $\mathrm{RH}$ values, the comparison of equilibrium moisture content capabilities according to temperature and $\mathrm{RH}$ [5,6], the consideration of the effect of material $\mathrm{pH}$ on fungi [7], and, although not applicable to internally insulated systems, the influence of UV exposure to inhibit fungal germination [8]. Additionally, terms of the equation were organized to maximise the output metric for materials which limit the likelihood of fungal viability. Under the intended composition of the equation, the contribution of each term to the total metric can be quantified [4].

Furthering this research could potentially improve the insulation installation process by strengthening the standards which govern it, increase the projected lifespan of materials installed, and bolster the trust of individuals considering investing in efficiency improvements ensuring that their money will be returned with increased value to their wallet and the environment.

\section{Conclusions}

Current standards which regulate the risk of fungal development lack protocols for transient conditions. Future studies should capitalize on the work initiated in this study to incorporate a more detailed understanding of factors that affect fungal viability in pursuit of a framework for understanding as well as comparing the performance of IWI following the exceedances of critical RH limits.

\section{References}

[1] HM Government (2010) Approved Document F- Ventilation.

[2] Marincioni, V. (2015) Moisture Risk Assessment for Internal Wall Insulation in Solid Wall Buildings in Bristol, Technical Report, Sustainable Traditional Buildings Alliance (unpublished).

[3] Chalabi, Z. (2019) A measure of resilience of building material to mould growth (unpublished).

[4] Hannah, N. (2020) Rethinking fungal risk to asses post-failure resilience of internal wall insulation systems, MSc Dissertation, UCL IEDE, London, UK.

[5] Lamond, W. J. and Graham, R. (1993) 'The Relationship Between the Equilibrium Moisture Content of Grass Mixtures and the Temperature and Humidity of the Air', Journal of Agricultural Engineering Research, 56(4), pp. 327-335. doi: 10.1006/jaer.1993.1083.

[6] Lawrence, M. G. (2005) The Relationship between Relative Humidity and the Dewpoint Temperature in Moist Air A Simple Conversion and Applications. Available at: http://journals.ametsoc.org/bams/article-pdf/86/2/225/3931558/bams-86-2-225.pdf (Accessed: 23 June 2020).

[7] Sedlbauer, K. (2001) Prediction of mould fungus formation on the surface of and inside building components.

[8] Norros, V. et al. (2015) 'Spore sensitivity to sunlight and freezing can restrict dispersal in wood-decay fungi', Ecology and Evolution. John Wiley and Sons Ltd, 5(16), pp. 3312-3326. doi: 10.1002/ece3.1589. 